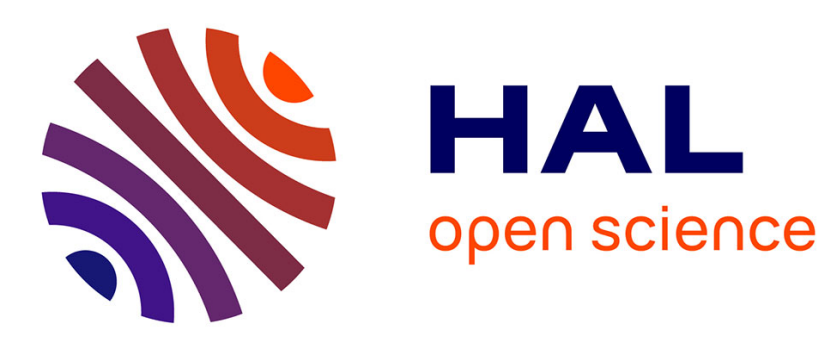

\title{
Analysis of the mechanical stresses on a squirrel cage induction motor by the finite element method
}

\author{
C. H. Jun, Alain Nicolas
}

\section{To cite this version:}

C. H. Jun, Alain Nicolas. Analysis of the mechanical stresses on a squirrel cage induction motor by the finite element method. IEEE Transactions on Magnetics, 1999, 35 (3 Part 1), pp.1282-1285. hal-00141539

\author{
HAL Id: hal-00141539 \\ https://hal.science/hal-00141539
}

Submitted on 18 Apr 2007

HAL is a multi-disciplinary open access archive for the deposit and dissemination of scientific research documents, whether they are published or not. The documents may come from teaching and research institutions in France or abroad, or from public or private research centers.
L'archive ouverte pluridisciplinaire HAL, est destinée au dépôt et à la diffusion de documents scientifiques de niveau recherche, publiés ou non, émanant des établissements d'enseignement et de recherche français ou étrangers, des laboratoires publics ou privés. 


\title{
Analysis of the Mechanical Stresses on a Squirrel Cage Induction Motor by the Finite Element Method
}

\author{
Chang-Hoon Jun and Alain Nicolas \\ CEGELY, UPRESA CNRS 5005, Ecole Centrale de Lyon, 69131 Ecully, France
}

\begin{abstract}
The mechanical deformations and stresses have been analyzed by the Finite Element Method (FEM) in 3 dimensions on the rotor bars of a small squirrel cage induction motor. We considered the magnetic forces and the centrifugal forces as sources which provoked the deformations and stresses on the rotor bars. The mechanical calculations have been performed after doing the electromagnetic Finite Element modeling on the motor in steady states with various slip conditions.
\end{abstract}

Index terms-Finite Element Analysis, magnetic forces, mechanical deformations and stresses, induction motor.

\section{INTRODUCTION}

In the squirrel cage induction motors, the torque is obtained by the reaction of the eddy currents in the rotor bars which are generated by the rotating magnetic fields in the stator windings. The magnetic force density is generated in the rotor bars by the eddy current density and the magnetic flux density $\left(\boldsymbol{F}=\boldsymbol{J}^{*} \boldsymbol{B}\left[\mathrm{N} / \mathrm{m}^{3}\right]\right)$. Therefore, the rotor bars suffer not only the centrifugal force but also the magnetic forces. The rotor bars are constructed commonly in aluminum by foundry process. The bars are considered as one of the most fragile parts in the squirrel cage induction motor because of the material, geometric characteristics and the hard load conditions [1]. By this fact, the service life of the motors can be prolonged by more careful fabrication and enhanced design. We want to offer the information of the stress states on the rotor bars that are useful to motor design engineers.

For the first step, to obtain electromagnetic parameters, we chose one squirrel cage induction motor that supplied $5.5 \mathrm{~kW}$ of output power, and then we carried out Finite Element modeling process on it by FLUX2D, a commercial program. Secondly, to calculate the magnetic forces, we have calculated the eddy current density $(\boldsymbol{J})$ and the magnetic flux density $(\boldsymbol{B})$ in the rotor bars from the magnetic vector potential values $(A)$ which have been given for final output values in FLUX2D. Finally, to analyze mechanical deformations and stresses, we have coded a FEM program in 3 dimensions.

\section{MODELING OF A SQUIRREL CAGE MOTOR IN 2-D}

The selected motor has 4 magnetic poles and it is supplied by $230 / 400 \mathrm{~V}$ three-phased tensions with $50 \mathrm{~Hz}$ and it has $0.3 \mathrm{~mm}$ of airgap thickness and $110 \mathrm{~mm}$ inner diameter of stator with $165 \mathrm{~mm}$ of effective rotor length. The stator has 36 winding slots and the rotor has 32 conductor slots.

Manuscript received June 3, 1998. revised March 1, 1999

C. H. Jun, (1) 609243 2204, fax (1) 609243 3030, cjun@pppl.gov

A. Nicolas, (33) 47218 6096, fax 7843 3717, nicolas@trotek.ec-lyon.fr
The windings of stator are constituted in single layer. The modeling with the aid of FLUX2D have been performed in steady states for various slip values. By the grace of the symmetry, only a quarter of the motor has been taken for the solution domain.

In FLUX2D which is a program for the analysis of electromagnetic field problems by Finite Element Method in 2 dimensions, the stator head windings and rotor rings of the motor can be considered in electric circuits that are linked the Finite Element solution domain. We have inserted the inductances and the resistances in this electric circuits after analytic calculation of these values. The iron core sheets of stator and rotor have been considered to have the non linear behavior of $\boldsymbol{H}-\boldsymbol{B}$. The results of the modeling are compared with the measured values for various slip conditions. This results are well concurrent with the measured values of the selected motor in maximum $10 \%$ difference.

\section{CALCULATION OF THE MAGNETIC FORCES}

\section{A. Calculation of the Magnetic Flux Density (B)}

To obtain magnetic forces, we have firstly calculated the magnetic flux density in rotor bars from the values of the magnetic vector potential $(A)$ that are the final results data in the output file of FLUX2D. The values of $\boldsymbol{A}$ are given by two components in FLUX2D - real and imaginary values - for a given phase on every node. The $\boldsymbol{A}$ is phasor that turns in complex plan according to the phase value.

Otherwise, the program of FLUX2D uses the quadratic triangular elements of 6 nodes. The vector potentials in two dimensions have only $\mathrm{z}$ components that are a function of the coordinates of $x-y$. The values of magnetic flux density are calculated element by element by the following equations :

$$
\begin{aligned}
& B=\operatorname{curl}(A)=i \frac{\partial A}{\partial y}-j \frac{\partial A}{\partial x}=i B_{x}+j B_{y} \\
& A: \text { vector potential } A=k A_{z}(x, y, t) \\
& B: \text { flux density } B=i B_{x}(x, y, t)+j B_{y}(x, y, t) \\
& i, j, k \text { : unit vectors }
\end{aligned}
$$

$$
\begin{aligned}
& B_{x}\left(x_{k}, y_{k}\right)=\sum_{i=1}^{6} \frac{\partial N_{i}\left(x_{k}, y_{k}\right)}{\partial y} \cdot A_{i}, k=1, \ldots, 6 \\
& B_{y}\left(x_{k}, y_{k}\right)=\sum_{i=1}^{6} \frac{-\partial N_{i}\left(x_{k}, y_{k}\right)}{\partial x}, A_{i}, k=1, \ldots, 6
\end{aligned}
$$

$x_{k}, y_{k}$ : coordinates of node $k$ in an element.

$N_{i}$ : shape functions of quadratic triangular element 


\section{B. Calculation of the eddy current density (J)}

We have also reckoned the eddy current density in rotor bars from the values of the vector potential by the following equations :

$$
\Delta-\operatorname{grad}(V)-\frac{\partial A}{\partial t}
$$

$V$ : electric potential

$J=\sigma E$

$\sigma$ : electric conductivity

Therefore,

$$
J=-\sigma \frac{\partial A}{\partial t}-\sigma \operatorname{grad}(V)
$$

For steady state,

$$
\begin{aligned}
& J=-j s \omega \sigma A-\sigma \operatorname{grad}(V) \\
& s: \operatorname{slip}(0<s<1) \\
& \omega: \text { angular frequency }\left(=2 \pi^{*} 50\right)
\end{aligned}
$$

The term of $\operatorname{grad}(V)$ is concerned with electric circuit which is composed of the short-circuited rotor bars[2], and at a given moment, its value is constant in the whole section area of each rotor bar in the solution domain. We have calculated this value from the data of post processor of FLUX2D.

\section{Calculation of the magnetic force density $(F)$}

The magnetic force density is calculated by the vector product between the eddy current and the magnetic flux density $\left(\boldsymbol{F}=\boldsymbol{J}^{*} \boldsymbol{B}\right)$ at every node. The values of this force have the dimension of $\left[\mathrm{N} / \mathrm{m}^{3}\right]$ and this force is body force that acts on the whole volume as centrifugal or gravity force.

The magnitude of $\boldsymbol{J}, \boldsymbol{B}$ and $\boldsymbol{F}$ are repeated according to rotor angle and time. The magnitude of $\boldsymbol{J}$ and $\boldsymbol{B}$ are dependent not on the $\boldsymbol{A}$ but on the derivative of $\boldsymbol{A}$. Therefore, these values reach maximum when turning potential vector $A$ is on imaginary axis, i.e. when the $\boldsymbol{A}$ has minimum real component. The $\boldsymbol{F}$ has also maximum value when $\boldsymbol{A}$ are on imaginary axis for a given node point.

Because the studied motor has 4 magnetic poles, $\boldsymbol{J}$ and $\boldsymbol{B}$ have a spatial periodic characteristics of $\cos (2 \theta)$ around the rotor axis. For the magnetic force density $\boldsymbol{F}$, it is obtained by the product of $\boldsymbol{J}$ and $\boldsymbol{B}$, and therefore the $\boldsymbol{F}$ has the periodic characteristic of $\cos (4 \theta)$ - four times repeated by one turn round the rotor axis[3].

The magnetic forces in the bars have greater values on the regions having greater radius for $\boldsymbol{B}$ increases according to rotor radius. The magnitude of $\boldsymbol{F}$ in the rotor bars increases, of course, in the function of the slip values owing to the augmentation of the eddy currents. We present two following figures that show the variation of the magnetic forces in the function of rotor angle and rotor radius. In these figures, we have compared the magnitudes of magnetic force density with centrifugal force.

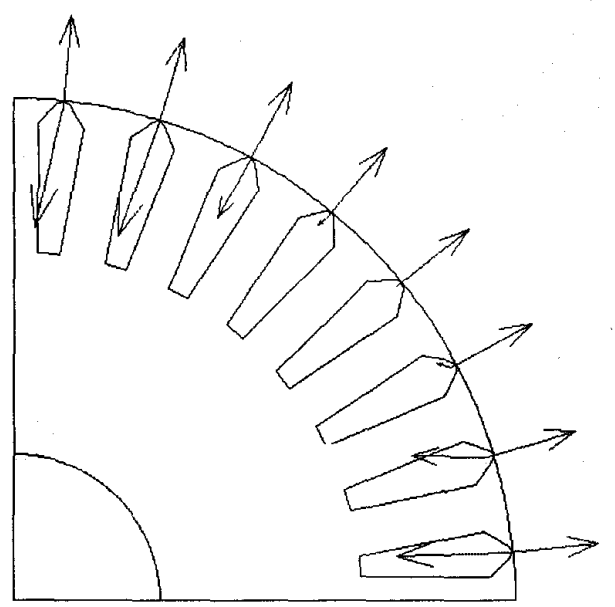

Fig.1 The distribution of the magnetic and centrifugal forces on the near regions to the airgap (1383 rpm, $7.8 \%$ slip)

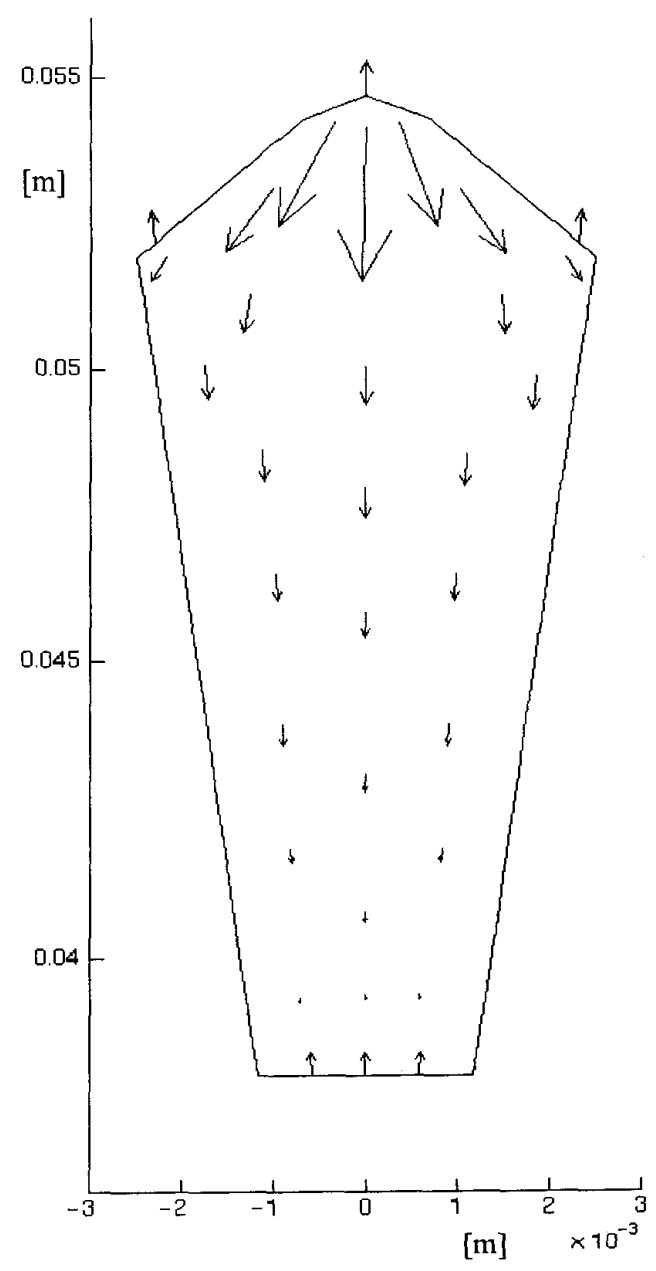

Fig. 2 The distribution of the magnetic forces in one rotor bar at the moment the forces become maximum, compared with centrifugal forces on the sides of the bar (1200rpm, 20\% slip) 
The rotor bars have long shape in radial direction to cut much more magnetic flux. The magnetic flux in the rotor prefer to pass the bars through the shortest path because aluminum bars have lower magnetic permeability than the iron core. Therefore, the flux pass the bars mainly in tangential direction of rotor axis. The magnetic forces have perpendicular direction to the flux path. And so, their radial components are much greater than the tangential components. By the direction of $\boldsymbol{J}$ and $\boldsymbol{B}$, the magnetic forces have the centripetal direction instead of centrifugal direction, with a little tangential component, and the maximum magnitude of magnetic forces is greater than that of centrifugal forces in the driving conditions having more little r.p.m. than the nominal angular velocity (1430 r.p.m.).

\section{Analysis of the mechanical stresses in 3-D}

\section{A. Solution domain and Boundary conditions}

The stresses in the rotor bars are mainly provoked by their magnetic forces and centrifugal forces. The mechanical stresses have been analyzed for only one selected rotor bar because all of the bars suffer very similar load conditions. The created FEM programs in 3 dimensions have been applied to calculate the stresses at the moment that the magnetic forces reach maximum magnitude.

For boundary conditions according to the $z$ axis, we assumed that the bar was completely fixed at one end $(z=0$, end ring side) and it had symmetric plan at $z=20 \mathrm{~mm}$. Actually, the rotor has the symmetric plan at $z=82.5 \mathrm{~mm}$ for the rotor length is $165 \mathrm{~mm}$. To save the memory space and the calculation time, we have shortened the solution domain according to $z$ axis from $82.5 \mathrm{~mm}$ to $20 \mathrm{~mm}$ after verifying it. The calculations of the verification that have been performed for various $z$ axis lengths assured us that the maximal stress on shortened bar from $82.5 \mathrm{~mm}$ until to $20 \mathrm{~mm}$ was not much different (10\% overvalued) than that on the full length bar. For boundary conditions in the $x-y$ plan, we assumed that the bar contracted only in the rotor slot or slided along slot walls and the iron core was solid infinitely, and not deformed.

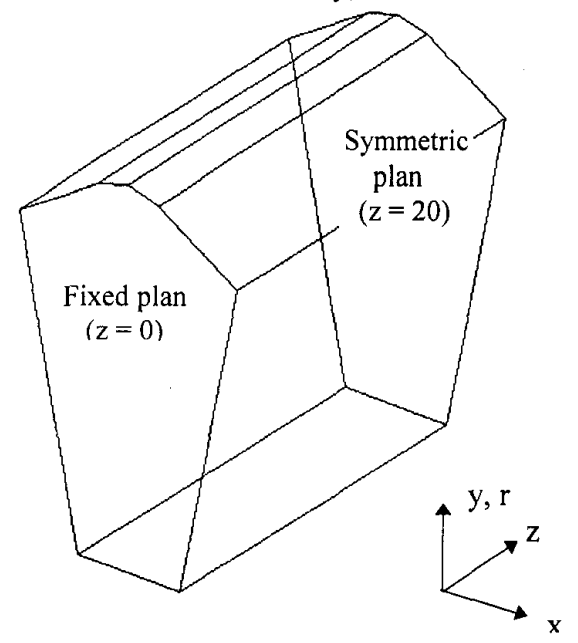

Fig. 3 The solution domain of the rotor bar in 3 dimensions for the analysis of the mechanical stresses

\section{B. Formulation}

We used the displacement method, for FE formulation. In this method, the displacement values on every node are firstly obtained and these values are to be derived to obtain the stresses values.

From the minimum potential energy principle on the elastic body that suffers only the body forces, the equations of FE formulation in 3 dimensions, on a node $q$ in any one element, are given as follows:

$$
\begin{array}{cc}
3 * 3 & 3 * 1 \\
{[K]^{q p}} & \{\delta\}^{q}=\left\{{ }^{3 * 1}\right.
\end{array}
$$

where,

$$
[K]^{3 * 3}=\iiint_{V(e)}[B]_{p}^{3^{* * 6}}\left[{ }^{T(e)}[]^{6^{* 6}}\right]^{(e)}[B]_{q}^{(e)} d V^{(e)}
$$

: stiffness matrix on the node $q$

$$
\{F\}^{q}=\iiint_{V(e)} N_{q}(x, y, z)\left\{F^{*}\right\}_{q}^{(e)} d V^{(e)}(10)
$$

: force vector on the node $q$.

$N_{q}$ : shape functions

$F^{*}$ : given body forces (known values)

$$
[B]_{q}^{(e)}=\left[\begin{array}{ccc}
\frac{\partial N_{q}}{\partial x} & 0 & 0 \\
0 & \frac{\partial N_{q}}{\partial y} & 0 \\
0 & 0 & \frac{\partial N_{q}}{\partial z} \\
\frac{\partial N_{q}}{\partial y} & \frac{\partial N_{q}}{\partial x} & 0 \\
0 & \frac{\partial N_{q}}{\partial z} & \frac{\partial N_{q}}{\partial y} \\
\frac{\partial N_{q}}{\partial z} & 0 & \frac{\partial N_{q}}{\partial x}
\end{array}\right], \quad q=1,2, \ldots \ldots, r
$$

: matrix relating strains and displacements (matrix concerning with the differentiation)

$$
[C]=E\left[\begin{array}{cccccc}
1-v & v & v & 0 & 0 & 0 \\
v & 1-v & v & 0 & 0 & 0 \\
v & v & 1-v & 0 & 0 & 0 \\
0 & 0 & 0 & \frac{1-2 v}{2} & 0 & 0 \\
0 & 0 & 0 & 0 & \frac{1-2 v}{2} & 0 \\
0 & 0 & 0 & 0 & 0 & \frac{1-2 v}{2}
\end{array}\right]
$$

$[C]$ : constitutive matrix (matrix relating strains and stresses) 


$$
E=\frac{E}{(1+v)(1-2 v)}
$$

(E : Young's modulus, $v$ : Poisson's ratio)

We adopted the prismatic element that is quadratic in $x-y$ plan ( 6 nodes) but cubic according to $\mathrm{z}$ axis (4 layers). One element has 24 nodes (therefore, $r$ in the equation (11) is 24) [4].

\section{Results and Discussion}

\section{A. Deformation of the bar in the rotor slot}

We have presented two case of the deformation of the bar in the following figures. In the case of nominal function of the motor $(1430 \mathrm{rpm})$, the resultant centrifugal forces are dominant and the bar deforms to the centrifugal direction. On the contrary, in the case of blocked rotor condition $(0 \mathrm{rpm})$, or at the early starting time in real service situation, the resultant magnetic forces are dominant and the bar deforms to the centripetal direction (see Figure 6).

We have calculated the deformation of the bar only when the magnetic forces reach their maximum. Actually, for the driving condition at the early starting time, the bars will be pulled to the centripetal direction by the magnetic forces and relaxed due to the variation of the magnetic forces. But in the nominal driving condition, the bars will be always pushed to the centrifugal direction due to dominant constant centrifugal force. In the middle velocity condition (e.g. $1000 \mathrm{rpm}$ ), or on the acceleration stage before arriving at the steady state for real service situation, the bars will be pushed to the centrifugal direction and pulled to the centripetal direction because the magnetic forces vary between zero and greater magnitude than the constant centrifugal forces.
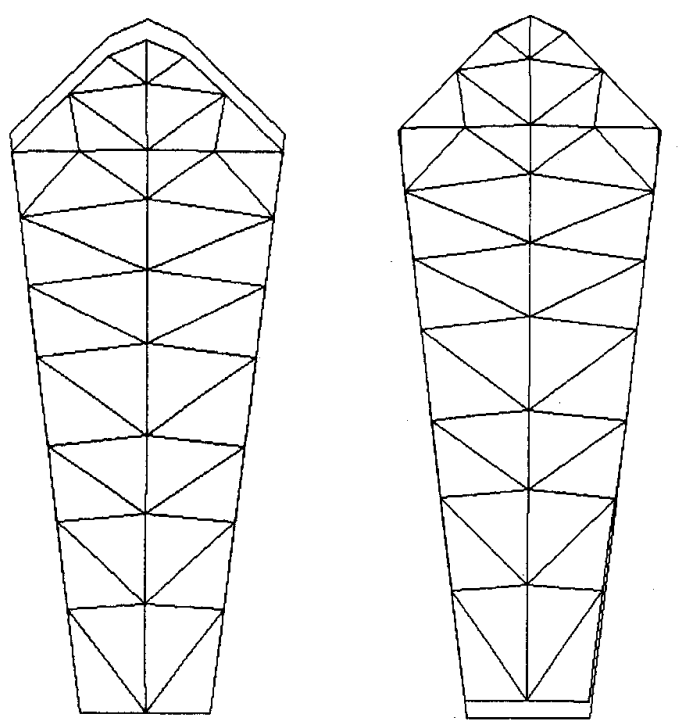

Fig. 6 Deformation of the rotor bar in $x-y$ plan at $z=20 \mathrm{~mm}$ : for the blocked rotor condition (left) and for the nominal velocity (right) when the magnetic forces reach maximum. (Deformations are exaggerated for easy presentation)

\section{B. Mechanical Stresses in the Rotor Bar}

The stress values on every node are obtained by differentiation, in each element, on the already calculated deformation values. This calculation is performed by the same principle as the equation (2), (3) to obtain the flux density $\boldsymbol{B}$ from the vector potential $A$.

\% The stresses have the greatest values not in the high velocity conditions but on the blocked rotor condition, or at the beginning of the starting. The integrated magnetic force in one bar at the beginning of starting stage is about 10 times greater than the integrated centrifugal forces at nominal function.

In the case of the starting stage, the maximum stress happens on the point having the greatest radius $(y$, or $r=54.7$ $\mathrm{mm})$ and being located at the end of the bar $(z=0)$ that is connected with the end ring. This stress level exceeds the fatigue limit of aluminum. Therefore, the service life of the motor will be diminished by the frequent starting, or the motor suffers more severe load condition for the service that demands more frequent starting.

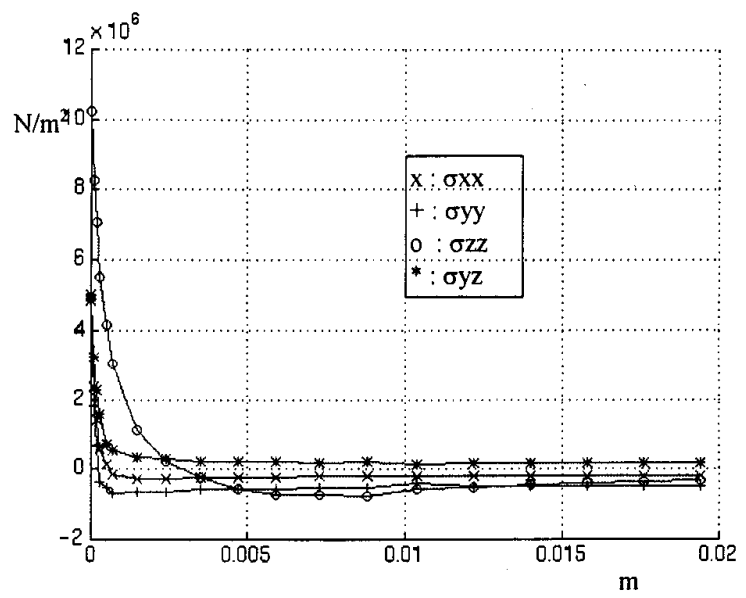

Fig. 7 The distribution of the stresses on the summit of the bar $(r=54.7 \mathrm{~mm})$ according to the longitudinal length $(\mathrm{z})$ at the beginning of the starting of the motor.

\section{REFERENCES}

[1] J.H. Dymond and R.D. Findlay, "Some commentary on the choice of rotor barre material for induction motor", IEEE Transactions on energy conversion, Vol. 10, No. 3, September 1995

[2] P. Lombard et G. Meunier, "Couplage des équations électriques et magnétiques," Journal de physique III France 3, pp.397-411, March 1993.

[3] S.J. Salon, M.J. DeBortoli and R. Palma, "Coupling of transient fields, circuits, and motion using finite element analysis," Journal of electromagnetic waves and applications, vol. 4, No. 11, pp. 1077-1106, 1990.

[4] P.P. Silvester and R.L. Ferrari, "Finite elements for electrical engineers," Cambridge university press, 1990. 